\title{
SYNTHESIS AND STRUCTURE OF IRIDIUM COMPLEX $\left[\mathrm{Ph}_{4} \mathrm{Sb}(\mathrm{DMSO})\right]^{+}\left[\mathrm{IrCl}_{4}\left(\mathrm{DMSO}_{2}\right]^{-}\right.$
}

\author{
V.V. Sharutin, vvsharutin@rambler.ru \\ O.K. Sharutina, sharutinao@mail.ru \\ V.S. Senchurin, senvl@rambler.ru \\ South Ural State University, Chelyabinsk, Russian Federation
}

\begin{abstract}
Iridium complex $\left[\mathrm{Ph}_{4} \mathrm{Sb}(\mathrm{DMSO})\right]^{+}\left[\mathrm{IrCl}_{4}(\mathrm{DMSO})_{2}\right]^{-}$(1) has been synthesized by interaction of tetraphenylstibonium chloride with sodium hexachloroiridate(III) in water. From Xray diffraction analysis data, two types of crystallographically independent cations $\left[\mathrm{Ph}_{4} \mathrm{Sb}(\mathrm{DMSO})\right]^{+}$and anions $\left[\mathrm{IrCl}_{4}(\mathrm{DMSO})_{2}\right]^{-}$exist in the crystal. The antimony atoms in cations have distorted trigonal-bipyramidal surroundings with $O$-dimethyl sulphoxide ligand in axial position (equatorial angles CSbC 113.6(2) ${ }^{\circ}-121.2(2)^{\circ}$, axial angles CSbO 178.33(18) ${ }^{\circ}$, $176.47(18)^{\circ}$, bond lengths $\mathrm{Sb}-\mathrm{C}_{\mathrm{eq}} 2.106(6)-2.119(6) \AA, \mathrm{Sb}-\mathrm{C}_{\mathrm{ax}} 2.126(6), 2.130(6) \AA, \mathrm{Sb}-\mathrm{O}$ $2.545(4), 2.465(4) \AA)$. In mononuclear octahedral anions the equatorial positions are occupied by chlorine atoms (angles ClIrCl-trans $178.69(7)^{\circ}-179.56(7)^{\circ}$, SIrS-trans $178.35(7)^{\circ}$, 175.88(7) ${ }^{\circ}$; dimethyl sulfoxide ligands are coordinated with iridium atoms through sulfur atoms (Ir-S 2.2958(16)-2.3148(17) $\AA$ ), bond lengths $\mathrm{Ir}-\mathrm{Cl}$ vary in the range 2.3407(19)2.3681(17) $\AA$. The structural organization in the crystal is determined by interionic hydrogen bonds $(\mathrm{H} \cdots \mathrm{Cl} 2.80-2.94 \AA$ and $\mathrm{H} \cdots \mathrm{O} 2.56-2.71 \AA)$.

Keywords: tetraphenylstibonium chloride, sodium hexachloroiridate(III), dimethyl sulfoxide, (dimethylsulfoxido)tetraphenylstibonium bis(dimethylsulfoxido)tetrachloroiridate(III), synthesis, X-ray diffraction analysis, structure.
\end{abstract}

\section{Introduction}

Ionic complex compounds of iridium with anions of the type $\left[\operatorname{IrHal}_{4}(S-\mathrm{DMSO})_{2}\right]^{-}$are described in the literature as single examples [1-4]. Note that both organic cations and tetraorganylphosphonium cations [4] can act as counterions in the crystals of these compounds, at this the structural organization of iridium complexes is determined by the presence of solvent molecules in their structure. In the present paper the synthesis of (dimethylsulfoxido)tetraphenylstibonium bis(dimethylsulfoxido)tetrachloroiridate (III), the characteristic features of its structure are examined.

\section{Results and Discussion}

In order to synthesize new iridium complexes we studied the reactions of tetraphenylstibonium chloride with sodium hexachloroiridate(III) in aqueous solution.

It has been found that after addition of sodium hexachloroiridate(III) in water to the aqueous solution of tetraphenylstibonium chloride in equimolar amount, followed by solvent evaporation and recrystallization of the solid precipitate from dimethyl sulfoxide, yellow crystals are formed; it is (dimethylsulfoxiodo)tetraphenylstibonium bis(dimethylsulfoxido)tetrachloroiridate(III) $\left[\mathrm{Ph}_{4} \mathrm{Sb}^{+}\left[\mathrm{IrCl}_{4}(\mathrm{DMSO})_{2}\right]^{-}\right.$(1):

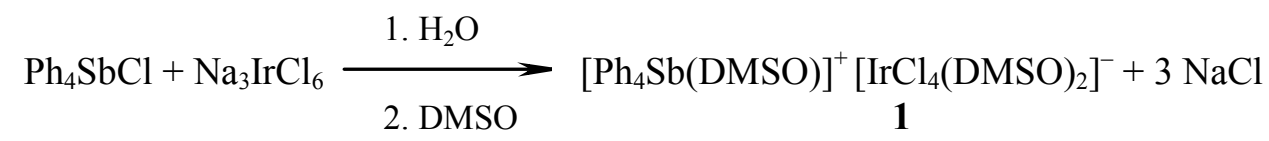

According to X-ray diffraction analysis, two types of crystallographically independent cations $\left[\mathrm{Ph}_{4} \mathrm{Sb}(\mathrm{DMSO})\right]^{+}$and anions $\left[\mathrm{IrCl}_{4}(\mathrm{DMSO})_{2}\right]^{-}$coexist in the crystal. The antimony atoms in cations have distorted trigonal-bipyramidal surroundings with $O$-dimethyl sulphoxide ligand in axial position (equatorial angles CSbC 113.6(2) $-121.2(2)^{\circ}$, axial angles CSbO 178.33(18) $)^{\circ}, 176.47(18)^{\circ}$, bond lengths $\mathrm{Sb}-$ $\mathrm{C}_{\mathrm{eq}}$ 2.106(6)-2.119(6) $\left.\AA, \mathrm{Sb}-\mathrm{C}_{\mathrm{ax}} 2.126(6), 2.130(6) \AA ̊, \mathrm{Sb}-\mathrm{O} 2.545(4), 2.465(4) \AA ̊\right)$ (Fig. 1). 


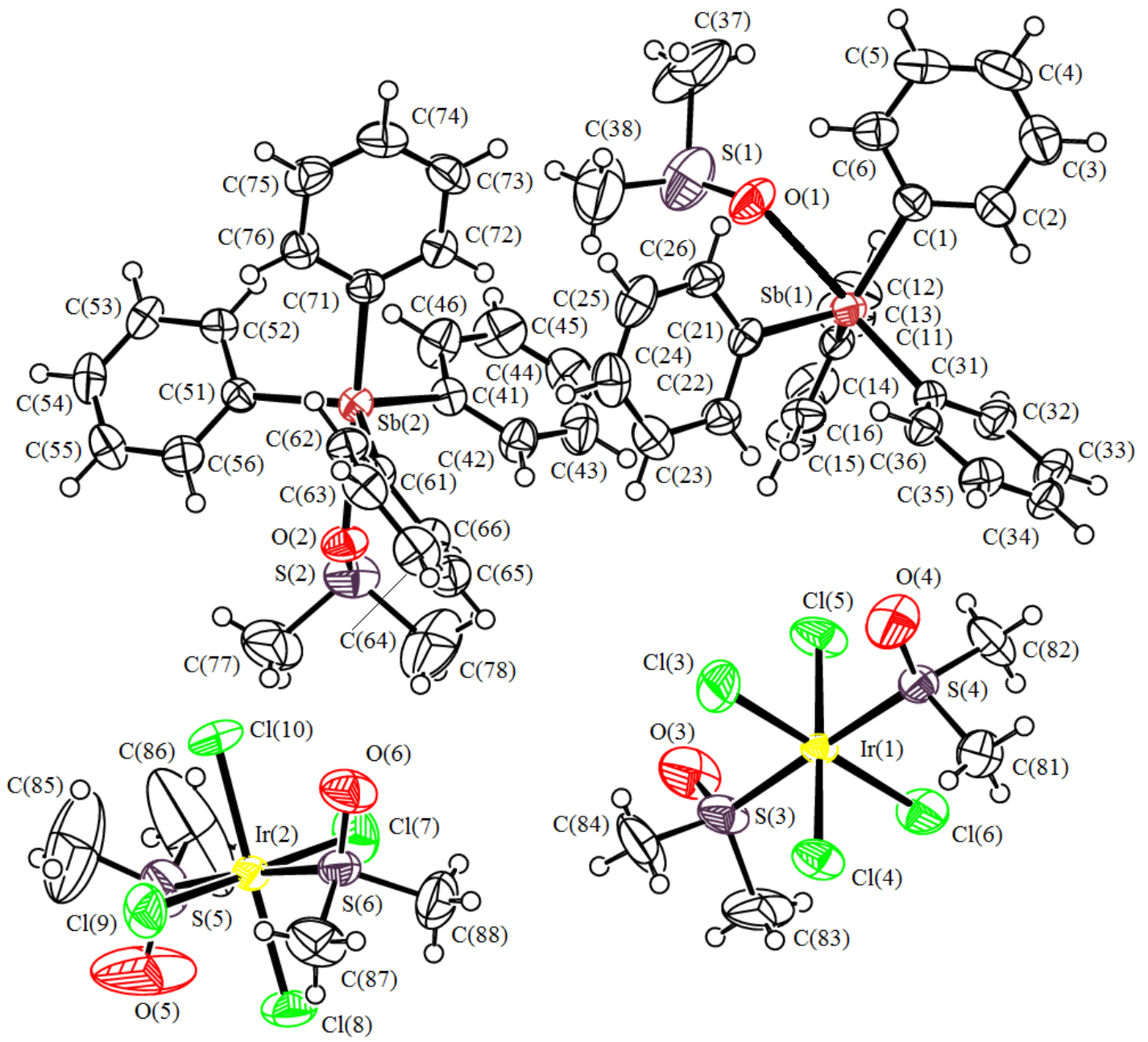

Fig. 1. The structure of compound 1

In mononuclear octahedral anions the equatorial positions are occupied by chlorine atoms (angles ClIrCl-trans $178.69(7)^{\circ}-179.56(7)^{\circ}$, SIrS-trans $178.35(7)^{\circ}, 175.88(7)^{\circ}$; dimethyl sulfoxide ligands are coordinated with iridium atoms through sulfur atoms (Ir-S 2.2958(16)-2.3148(17) $\AA$ ), bond lengths Ir-Cl vary in the range 2.3407(19)-2.3681(17) $\AA$. The structural organization in the crystal is determined by interionic hydrogen bonds $(\mathrm{H} \cdots \mathrm{Cl} 2.80-2.94 \AA$ and $\mathrm{H} \cdots \mathrm{O} 2.56-2.71 \AA)$, the strongest ones are shown in Fig. 2. Cations and anions are packed in stacks oriented along crystallographic axis $a$.

X-ray diffraction analysis of the complex 1 crystal was performed on the Bruker D8 QUEST automatic four-circle diffractometer (Mo $\mathrm{K}_{\alpha}$-emission, $\lambda=0.71073 \AA$, graphite monochromator). The data were collected and analyzed, the unit cell parameters were refined, and the absorption correction was applied using the SMART and SAINT-Plus programs [5]. All calculations for structure determination and refinement were performed using the SHELXL/PC programs [6]. The structure 1 was determined by the direct method and refined by the least-squares method in the anisotropic approximation for nonhydrogen atoms. The main crystallographic data and refinement results for structure $\mathbf{1}$ are listed in Table 1, the selected bond lengths and bond angles are given in Table 2.

The full tables of atomic coordinates, bond lengths, and bond angles were deposited with the Cambridge Crystallographic Data Centre (CCDC 1048264; deposit@ccdc.cam.ac.uk; http://www.ccdc.cam.ac.uk). 


\section{Organometallic chemistry}

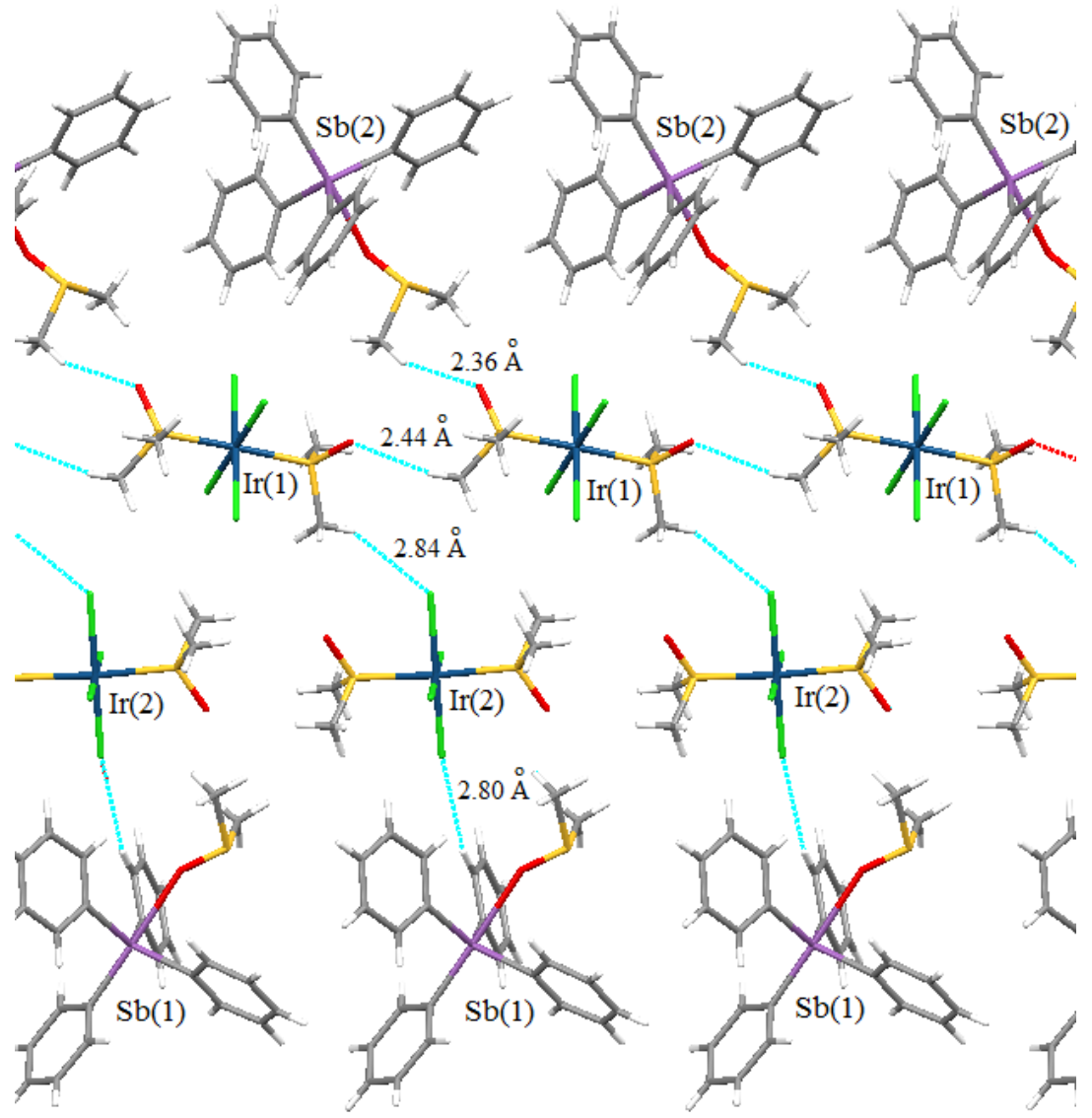

Fig. 2. Interionic bonds in the crystal of compound 1

Crystallographic data and the experimental and structure refinement parameters for compound 1

Table 1

\begin{tabular}{|c|c|}
\hline Parameter & Value \\
\hline Empirical formula & $\mathrm{C}_{60} \mathrm{O}_{6} \mathrm{~S}_{6} \mathrm{Cl}_{8} \mathrm{Sb}_{2} \mathrm{Ir}_{2} \mathrm{H}_{76}$ \\
\hline Formula weight & 1997.07 \\
\hline$T, \mathrm{~K}$ & $296(2)$ \\
\hline Crystal system & Triclinic \\
\hline Space group & $\mathrm{P}-1$ \\
\hline$a, \AA$ & $9.5963(6)$ \\
\hline$b, \AA$ & $19.2090(14)$ \\
\hline$c, \AA$ & $19.5688(14)$ \\
\hline$\alpha$, deg & $91.109(3)$ \\
\hline$\beta$, deg & $90.549(2)$ \\
\hline$\gamma$, deg & $90.095(3)$ \\
\hline$V, \AA^{3}$ & $3606.4(4)$ \\
\hline$Z$ & 2 \\
\hline$\rho($ calcd. $)$, g/cm & 1.839 \\
\hline$\mu, m^{-1}$ & 4.935 \\
\hline$F(000)$ & 1944.0 \\
\hline Crystal size, mm & $0.11 \times 0.18 \times 0.24$ \\
\hline Range of data collection, deg & $5.98-41.32^{\circ}$ \\
\hline Range of refraction indices & 67394 \\
\hline Measured reflections & 7363 \\
\hline Independent reflections & 0.0325 \\
\hline$R_{\text {int }}$ & $-9 \leq \mathrm{h} \leq 9,-19 \leq \mathrm{k} \leq 19,-19 \leq 1 \leq 19$ \\
\hline
\end{tabular}


Table 1 (end)

\begin{tabular}{|c|c|}
\hline Parameter & Value \\
\hline Refinement variables & 769 \\
\hline$G O O F$ & 1.026 \\
\hline$R$ factors for $\mathrm{F}^{2}>2 \sigma\left(\mathrm{F}^{2}\right)$ & $R_{1}=0.0237, w R_{2}=0.0533$ \\
\hline$R$ factors for all reflections & $R_{1}=0.0286, w R_{2}=0.0560$ \\
\hline Residual electron density (min/max), $e / \AA^{3}$ & $1.12 /-0.93$ \\
\hline
\end{tabular}

Selected bond lengths and bond angles in the structure of compound 1

Table 2

\begin{tabular}{|c|c|c|c|}
\hline Bond & $d, \AA$ & Angle & $\omega$, deg \\
\hline $\operatorname{Ir}(1)-S(3)$ & $2.2958(16)$ & $\mathrm{S}(3) \operatorname{Ir}(1) \mathrm{S}(4)$ & $178.35(7)$ \\
\hline $\operatorname{Ir}(1)-S(4)$ & $2.3002(15)$ & $\mathrm{S}(3) \operatorname{Ir}(1) \mathrm{Cl}(3)$ & $90.09(8)$ \\
\hline $\operatorname{Ir}(1)-\mathrm{Cl}(3)$ & $2.3407(19)$ & $\mathrm{S}(3) \operatorname{Ir}(1) \mathrm{Cl}(4)$ & $87.48(6)$ \\
\hline $\operatorname{Ir}(1)-\mathrm{Cl}(4)$ & $2.3529(16)$ & $\mathrm{S}(3) \operatorname{Ir}(1) \mathrm{Cl}(5)$ & $92.21(6)$ \\
\hline $\operatorname{Ir}(1)-\mathrm{Cl}(5)$ & $2.3500(17)$ & $\mathrm{S}(3) \operatorname{Ir}(1) \mathrm{Cl}(6)$ & $90.33(7)$ \\
\hline $\operatorname{Ir}(1)-\mathrm{Cl}(6)$ & $2.3681(17)$ & $\mathrm{S}(4) \operatorname{Ir}(1) \mathrm{Cl}(3)$ & $91.55(7)$ \\
\hline $\operatorname{Ir}(2)-S(5)$ & $2.311(2)$ & $\mathrm{S}(4) \operatorname{Ir}(1) \mathrm{Cl}(4)$ & $92.71(6)$ \\
\hline $\operatorname{Ir}(2)-S(6)$ & $2.3148(17)$ & $\mathrm{S}(4) \operatorname{Ir}(1) \mathrm{Cl}(5)$ & $87.62(6)$ \\
\hline $\operatorname{Ir}(2)-\mathrm{Cl}(7)$ & $2.3493(18)$ & $\mathrm{S}(4) \operatorname{Ir}(1) \mathrm{Cl}(6)$ & $88.03(6)$ \\
\hline $\operatorname{Ir}(2)-\mathrm{Cl}(8)$ & $2.3498(17)$ & $\mathrm{Cl}(3) \operatorname{Ir}(1) \mathrm{Cl}(4)$ & $90.30(8)$ \\
\hline $\operatorname{Ir}(2)-\mathrm{Cl}(9)$ & $2.3546(17)$ & $\mathrm{Cl}(3) \operatorname{Ir}(1) \mathrm{Cl}(5)$ & $88.89(8)$ \\
\hline $\operatorname{Ir}(2)-\mathrm{Cl}(10)$ & $2.3396(17)$ & $\mathrm{Cl}(3) \operatorname{Ir}(1) \mathrm{Cl}(6)$ & $179.56(7)$ \\
\hline $\mathrm{Sb}(1)-\mathrm{O}(1)$ & $2.545(4)$ & $\mathrm{Cl}(4) \operatorname{Ir}(1) \mathrm{Cl}(6)$ & $89.60(7)$ \\
\hline $\mathrm{Sb}(1)-\mathrm{C}(1)$ & $2.117(6)$ & $\mathrm{Cl}(5) \operatorname{Ir}(1) \mathrm{Cl}(4)$ & $179.14(7)$ \\
\hline $\mathrm{Sb}(1)-\mathrm{C}(11)$ & $2.106(6)$ & $\mathrm{Cl}(5) \operatorname{Ir}(1) \mathrm{Cl}(6)$ & $91.20(8)$ \\
\hline $\mathrm{Sb}(1)-\mathrm{C}(21)$ & $2.106(6)$ & $\mathrm{S}(5) \operatorname{Ir}(2) \mathrm{S}(6)$ & $175.88(7)$ \\
\hline $\mathrm{Sb}(1)-\mathrm{C}(31)$ & $2.126(6)$ & $\mathrm{S}(5) \operatorname{Ir}(2) \mathrm{Cl}(10)$ & $91.53(8)$ \\
\hline $\mathrm{Sb}(2)-\mathrm{O}(2)$ & $2.465(4)$ & $\mathrm{Cl}(7) \operatorname{Ir}(2) \mathrm{Cl}(9)$ & $179.22(7)$ \\
\hline $\mathrm{Sb}(2)-\mathrm{C}(51)$ & $2.106(6)$ & $\mathrm{Cl}(10) \operatorname{Ir}(2) \mathrm{Cl}(8)$ & $178.69(7)$ \\
\hline $\mathrm{Sb}(2)-\mathrm{C}(71)$ & $2.130(6)$ & $\mathrm{Cl}(10) \operatorname{Ir}(2) \mathrm{Cl}(9)$ & $90.30(8)$ \\
\hline $\mathrm{Sb}(2)-\mathrm{C}(41)$ & $2.111(6)$ & $\mathrm{C}(1) \mathrm{Sb}(1) \mathrm{O}(1)$ & 77.93(19) \\
\hline $\mathrm{Sb}(2)-\mathrm{C}(61)$ & $2.119(6)$ & $\mathrm{C}(1) \mathrm{Sb}(1) \mathrm{C}(31)$ & $100.4(2)$ \\
\hline $\mathrm{O}(4)-\mathrm{S}(4)$ & $1.459(5)$ & $\mathrm{C}(11) \mathrm{Sb}(1) \mathrm{O}(1)$ & 78.92(18) \\
\hline $\mathrm{O}(6)-\mathrm{S}(6)$ & $1.464(5)$ & $\mathrm{C}(11) \mathrm{Sb}(1) \mathrm{C}(1)$ & $116.7(2)$ \\
\hline $\mathrm{S}(1)-\mathrm{O}(1)$ & $1.457(5)$ & $\mathrm{C}(11) \mathrm{Sb}(1) \mathrm{C}(21)$ & $113.6(2)$ \\
\hline $\mathrm{S}(1)-\mathrm{C}(38)$ & $1.739(10)$ & $\mathrm{C}(11) \mathrm{Sb}(1) \mathrm{C}(31)$ & $102.2(2)$ \\
\hline $\mathrm{S}(1)-\mathrm{C}(37)$ & $1.785(11)$ & $\mathrm{C}(21) \mathrm{Sb}(1) \mathrm{O}(1)$ & $80.45(19)$ \\
\hline $\mathrm{S}(2)-\mathrm{O}(2)$ & $1.517(4)$ & $\mathrm{C}(21) \mathrm{Sb}(1) \mathrm{C}(1)$ & $119.2(2)$ \\
\hline $\mathrm{S}(2)-\mathrm{C}(77)$ & $1.763(8)$ & $\mathrm{C}(21) \mathrm{Sb}(1) \mathrm{C}(31)$ & $100.2(2)$ \\
\hline $\mathrm{S}(2)-\mathrm{C}(78)$ & $1.753(9)$ & $\mathrm{C}(31) \mathrm{Sb}(1) \mathrm{O}(1)$ & $178.33(18)$ \\
\hline $\mathrm{S}(3)-\mathrm{O}(3)$ & $1.450(5)$ & $\mathrm{C}(51) \mathrm{Sb}(2) \mathrm{O}(2)$ & $82.50(18)$ \\
\hline $\mathrm{S}(3)-\mathrm{C}(84)$ & $1.779(9)$ & $\mathrm{C}(51) \mathrm{Sb}(2) \mathrm{C}(71)$ & $98.7(2)$ \\
\hline $\mathrm{S}(3)-\mathrm{C}(83)$ & $1.758(8)$ & $\mathrm{C}(51) \mathrm{Sb}(2) \mathrm{C}(41)$ & $114.4(2)$ \\
\hline $\mathrm{S}(4)-\mathrm{C}(81)$ & $1.764(6)$ & $\mathrm{C}(51) \mathrm{Sb}(2) \mathrm{C}(61)$ & $121.2(2)$ \\
\hline$S(4)-C(82)$ & $1.769(7)$ & $\mathrm{C}(71) \mathrm{Sb}(2) \mathrm{O}(2)$ & $176.47(18)$ \\
\hline $\mathrm{S}(5)-\mathrm{C}(86)$ & $1.676(11)$ & $\mathrm{C}(41) \mathrm{Sb}(2) \mathrm{O}(2)$ & $83.41(18)$ \\
\hline $\mathrm{S}(5)-\mathrm{O}(5)$ & $1.477(6)$ & $\mathrm{C}(41) \mathrm{Sb}(2) \mathrm{C}(71)$ & $99.0(2)$ \\
\hline$S(5)-C(85)$ & $1.678(11)$ & $\mathrm{C}(41) \mathrm{Sb}(2) \mathrm{C}(61)$ & $117.3(2)$ \\
\hline $\mathrm{S}(6)-\mathrm{C}(88)$ & $1.765(7)$ & $\mathrm{C}(61) \mathrm{Sb}(2) \mathrm{O}(2)$ & $77.58(18)$ \\
\hline$S(6)-C(87)$ & $1.770(7)$ & $\mathrm{C}(61) \mathrm{Sb}(2) \mathrm{C}(71)$ & $99.0(2)$ \\
\hline
\end{tabular}




\title{
Organometallic chemistry
}

\section{Conclusion}

The structure of iridium complex $\left[\mathrm{Ph}_{4} \mathrm{Sb}(\mathrm{DMSO})\right]^{+}\left[\mathrm{IrCl}_{4}(\mathrm{DMSO})_{2}\right]^{-}$, obtained from tetraphenylstibonium chloride and sodium hexachloroiridate (III) in water, followed by recrystallization from dimethyl sulfoxide, has been established by X-ray diffraction analysis.

\section{References}

1. Haddad Y.M.Y., Henbest H.B., Trocha-Grimshaw J. Aspects of Catalysis. Part II. Dimethyl Sulphoxide Complexes of Iridium(III) Including Hydrides. Journal of the Chemical Society, Perkin Transactions 1, 1974, pp. 592-595.

2. Messori L., Marcon G., Orioli P, Fontani M., Zanello P., Bergamoc A., Savac G., Murad P. Molecular Structure, Solution Chemistry and Biological Properties of the Novel [ImH][trans$\left.\mathrm{IrCl}_{4}(\mathrm{Im})(\mathrm{DMSO})\right]$, (I) and of the Orange Form of 4 [(DMSO) $\left.{ }_{2} \mathrm{H}\right]\left[\right.$ trans- $\left.\mathrm{IrCl}_{4}(\mathrm{DMSO})_{2}\right]$, (II). Journal of Inorganic Biochemistry, 2003, vol. 95, iss. 1, pp. 37-46.

3. Albertí F.M., Fiol J.J., García-Raso A., Torres M., Terrón A., Barceló-Oliver M., Prieto M.J., Moreno V., Molins E. Ruthenium(III) and Iridium(III) Complexes with Nicotine. Polyhedron, 2010, vol. 29, iss. 1, pp. 34-41. DOI: 10.1016/j.poly.2009.05.082

4. Sharutin V.V., Sharutina O.K., Senchurin V.S., Somov N.V. [Synthesis and Structure of Iridium Complexes $\left[\mathrm{Ph}_{3} \mathrm{PR}\right]\left[\right.$ trans- $\left.\mathrm{IrCl}_{4}(\mathrm{DMSO})_{2}\right]$. Russian Journal of General Chemistry, 2015, vol. 85, no. 3, pp. 634-639. DOI: 10.1134/S1070363215030184

5. SMART and SAINT-Plus. Versions 5.0. Data Collection and Processing Software for the SMART System. - Bruker AXS Inc. - 1998. - Madison, Wisconsin, USA.

6. SHELXTL/PC. Versions 5.10. An Integrated System for Solving, Refining and Displaying Crystal Structures From Diffraction Data. - Bruker AXS Inc. -1998. - Madison, Wisconsin, USA.

Received 10 September 2015

УДК 547.243; 546.9; 548.312.2

DOI: $10.14529 /$ chem150410

\section{СИНТЕЗ И СТРОЕНИЕ КОМПЛЕКСА ИРИДИЯ $\left[\mathrm{Ph}_{4} \mathrm{Sb}(\mathrm{DMSO})\right]^{+}\left[\mathrm{IrCl}_{4}(\mathrm{DMSO})_{2}\right]^{-}$}

\author{
В.В. Шарутин, О.К. Шарутина, В.С. Сенчурин \\ Южно-Уральский государственный университет, г. Челябинск
}

Взаимодействием хлорида тетрафенилстибония с гексахлороиридатом(III) натрия в воде синтезирован комплекс иридия $\left[\mathrm{Ph}_{4} \mathrm{Sb}(\mathrm{DMSO})\right]^{+}\left[\mathrm{IrCl}_{4}(\mathrm{DMSO})_{2}\right]^{-}$(1). По данным PCA в кристалле присутствуют по два типа кристаллографически независимых катионов $\left[\mathrm{Ph}_{4} \mathrm{Sb}(\mathrm{DMSO})\right]^{+}$и анионов $\left[\mathrm{IrCl}_{4}(\mathrm{DMSO})_{2}\right]^{-}$. Атомы сурьмы в катионах имеют искаженное тригонально-бипирамидальное окружение с аксиально расположенным $O$-диметилсульфоксидным лигандом, (экваториальные углы CSbC 113.6(2) $-121.2(2)^{\circ}$,

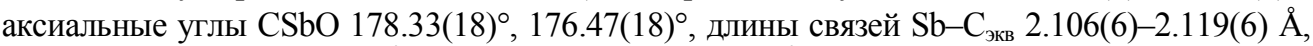
$\mathrm{Sb}-\mathrm{C}_{\text {акс }} 2.126(6), 2.130(6) \AA$, Sb-O 2.545(4), 2.465(4) $\AA$ ). В моноядерных октаэдрических анионах экваториальные положения занимают атомы хлора (углы ClIrCl-транс $178.69(7)^{\circ}-179.56(7)^{\circ}$, SirS-транс 178.35(7) ${ }^{\circ}, 175.88(7)^{\circ}$; диметилсульфоксидные лиганды координируются с атомами Ir посредством атомов серы (Ir-S 2.2958(16)-2.3148(17) А), длины связей $\mathrm{Ir}-\mathrm{Cl}$ изменяются в интервалах 2.3407(19)2.3681(17) А. Структурная организация в кристалле обусловлена межионными водо-

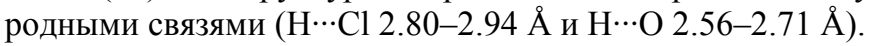

Ключевые слова: хлорид тетрафенилстибония, гексахлороиридат(III) натрия, диметилсульфоксид, бис(диметилсульфоксидо)тетрахлороиридат(III) (диметилсульфоксидо)тетрафенилстибония, синтез, рентгеноструктурный анализ, строение. 
Шарутин Владимир Викторович - доктор химических наук, профессор, старший научный сотрудник УНИД, Южно-Уральский государственный университет. 454080, г. Челябинск, пр. им. В.И. Ленина, 76. E-mail: vvsharutin@rambler.ru

Шарутина Ольга Константиновна - доктор химических наук, профессор, кафедра аналитической химии, Южно-Уральский государственный университет. 454080, г. Челябинск, пр. им. В.И. Ленина, 76. Е-mail: sharutinao@mail.ru

Сенчурин Владислав Станиславович - кандидат химических наук, кафедра органической химии, Южно-Уральский государственный университет. 454080, г. Челябинск, пр. им. В.И. Ленина, 76. E-mail: senvl@rambler.ru

Поступила в редакцию 10 сентября 2015 2.

\section{ОБРАЗЕЦ ЦИТИРОВАНИЯ}

Sharutin, V.V. Synthesis and structure of iridium complex $\left[\mathrm{Ph}_{4} \mathrm{Sb}(\mathrm{DMSO})\right]^{+}\left[\mathrm{IrCl}_{4}(\mathrm{DMSO})_{2}\right]^{-}$/ V.V. Sharutin, O.K. Sharutina, V.S. Senchurin // Вестник ЮУрГУ. Серия «Химия». - 2015. - T. 7, № 4. - C. 80-85. DOI $10.14529 /$ chem 150410

\section{FOR CITATION}

Sharutin V.V., Sharutina O.K., Senchurin V.S. Synthesis and Structure of Iridium Complex $\left[\mathrm{Ph}_{4} \mathrm{Sb}(\mathrm{DMSO})\right]^{+}\left[\mathrm{IrCl}_{4}(\mathrm{DMSO})_{2}\right]^{-}$. Bulletin of the South Ural State University. Ser. Chemistry. 2015, vol. 7, no. 4, pp. 80-85. DOI: $10.14529 /$ chem 150410 